\title{
MyWalk: A Mobile App for Gait Asymmetry Rehabilitation in the Community
}

\author{
Tuck-Voon How ${ }^{1}$, Justin Chee ${ }^{1,2}$, Eric Wan ${ }^{1,3}$, Alex Mihailidis ${ }^{1,2,4}$ \\ Institute of Biomaterials \& Biomedical Engineering ${ }^{1}$; Graduate Department of Rehabilitation Science ${ }^{2}$; \\ Edward S. Rogers Sr. Department of Electrical and Computer Engineering ${ }^{3}$; \\ Department of Occupational Science \& Occupational Therapy ${ }^{4}$ \\ University of Toronto; Toronto, Canada \\ \{tuckvoon.how, justin.chee, eric.wan, alex.mihailidis\}@ mail.utoronto.ca
}

\begin{abstract}
MyWalk is a mobile app to enable gait rehabilitation in the community. Particularly, it can be used to treat and assess step-time asymmetry (STA) - which occurs when the amount of time between consecutive heel strikes is uneven. Temporal gait asymmetry, of which STA is a form, is common post-stroke and could result in difficulties such as joint degeneration or musculoskeletal pain. By enabling STA rehabilitation on a smartphone, post-stroke patients can now improve and assess their STA within the context of their everyday environments. Initial validation of MyWalk's algorithm showed minimal error (RMSE 2.20-2.67\%) versus a foot-switch ground truth in detecting STA for symmetric walking conditions, but larger error (RMSE 13.82-16.34\%) in asymmetric walking conditions. Future work will be to improve the accuracy of MyWalk's STA algorithm.
\end{abstract}

Keywords-biofeedback; technologies; gait; outpatient rehabilitation; smartphones; stroke; step-time asymmetry.

\section{INTRODUCTION}

Stroke is a leading cause of death and disability within Canada. It is estimated that 315,000 (1.1\%) Canadians living in the community suffer from the effects of stroke [1], which includes deficits in physical, cognitive, or communication functions [2]. Rehabilitation after stroke is a key aspect in addressing these functional deficits.

A major focus of post-stroke rehabilitation is the restoration of walking, as this is one of the goals that is most frequently stated among stroke survivors [3]. The ability to walk again is linked with a recovery of independence. However, achieving a highly functional gait pattern requires significant physical rehabilitation and practice [4]. Alterations to a stroke patient's gait pattern must be made with respect to spatiotemporal parameters, kinematics/kinetics, and energy expenditure in order to improve gait to the level of a typical healthy adult [5].

An important gait parameter to target is temporal symmetry. One form of temporal asymmetry is when the amount of time between the heel strike of one foot and the consecutive heel strike of the contralateral foot is different for each leg of an individual. We refer to this as step-time asymmetry (STA). Overall, temporal gait asymmetry is problematic because it has been speculated to play a role in the development of joint degeneration, musculoskeletal pain, balance difficulties, inefficient energy use, and decreased activity levels [6]. Yet, if a stroke patient has the physical capacity to achieve a more normalized gait pattern; physical therapy and biofeedback training could be a means to treat and reduce the occurrence of STA. Examples of therapy to improve functional gait include: stimulating gait patterns through manipulations from a physical therapist during overground training sessions [7]; treadmill training [8]; and task-specific circuit training [9]. In addition, recent interventions have been developed that target gait asymmetry more directly with promising results. For example, Patterson demonstrated that active, real-time feedback during gait training has the potential to reduce asymmetry in stroke patients [5].

The challenge though is continuing such therapy outside of the hospital setting. Once patients are discharged from inpatient rehabilitation they are exposed to fewer opportunities to receive feedback on their gait patterns, and take part in less gait-specific training [10]. This in turn could lead to a deterioration of gait quality years after stroke [11]. Other challenges include ensuring that patients are motivated to continue therapy outside of the clinic, and for inpatient therapy benefits to continue into real-world settings [12].

Telerehabilitation is thought to be one way to improve outpatient care. By definition, telerehabilitation is the ability to conduct rehabilitative therapy, assessment, or training over a distance through the use of information and communication technology [13]. With the changing landscape towards a pervasive computing society, the possible ways of conducting telerehabilitation are also growing [14]. Mobile and wearable forms of computing now allow for rehabilitation to be more seamlessly embedded into an individual's everyday context. This has profound implications on gait rehabilitation within the community.

The purpose of this research is to explore how pervasive computing technology can be applied to gait asymmetry rehabilitation. Particularly, a mobile app was created to assess and reduce the occurrence of STA. The novelty of this technology is that it enables rehabilitation to be conducted within the context of a patient's everyday environment.

\section{SYSTEM DESCRIPTION}

\section{A. System Overview}

MyWalk is an Android app developed for rehabilitating STA in outpatient community settings. It was designed primarily for the stroke population. Previously, researchers have attempted to use smart phones to measure gait symmetry (e.g., [15-16]); however MyWalk expands upon this use case by incorporating biofeedback training and novel assessment 
strategies that can be used in the context of a patient's daily environment. Essentially, MyWalk attempts to expand the gait rehabilitation paradigm into the outpatient community setting.

The app has three modes of operation: (1) training; (2) community walk; and (3) progress history. In the training mode, the user/patient can conduct short (one minute), repetitive exercises to improve their temporal symmetry. With the community walk mode, the user can identify how different environments affect their temporal symmetry. Lastly, in the progress history, the user can track how their temporal symmetry has changed over time and share these results with his/her therapist.

\section{B. Step-time Asymmetry Calculation}

The foundation of MyWalk is the ability to monitor realtime STA in different environments. To do this, MyWalk utilizes a modified version of Yang's algorithm, which was developed to detect spatial gait symmetry from trunk acceleration [17]. Alterations to Yang's algorithm include: additional input signal conditioning to take into account varying accelerometer sampling rates and noise on Android devices, a faster update rate to provide real-time feedback, and an alteration of the spatial symmetry calculation in order to calculate temporal symmetry.

Android smartphones typically have a built in tri-axial accelerometer. To begin the analysis of a user's STA, the user must secure the phone to his/her trunk midline (i.e., at the level of the chest or waist). A user may do this either with a harness or by holding the smartphone to his/her body. When the user begins to walk, the MyWalk algorithm captures the vertical acceleration of trunk motion. For signal conditioning, accelerometer values are linearly interpolated and re-sampled at a frequency of $60 \mathrm{~Hz}$; in doing so autocorrelation can be performed at a later stage. Additionally, the acceleration values are passed through a $4^{\text {th }}$ order low-pass Butterworth filter with a cut-off frequency of $5 \mathrm{~Hz}$ to reduce spatial and temporal noise.

The output from the filter is then fed into a $3.5 \mathrm{~s}$ time window (approximately the time for six steps [17]) and
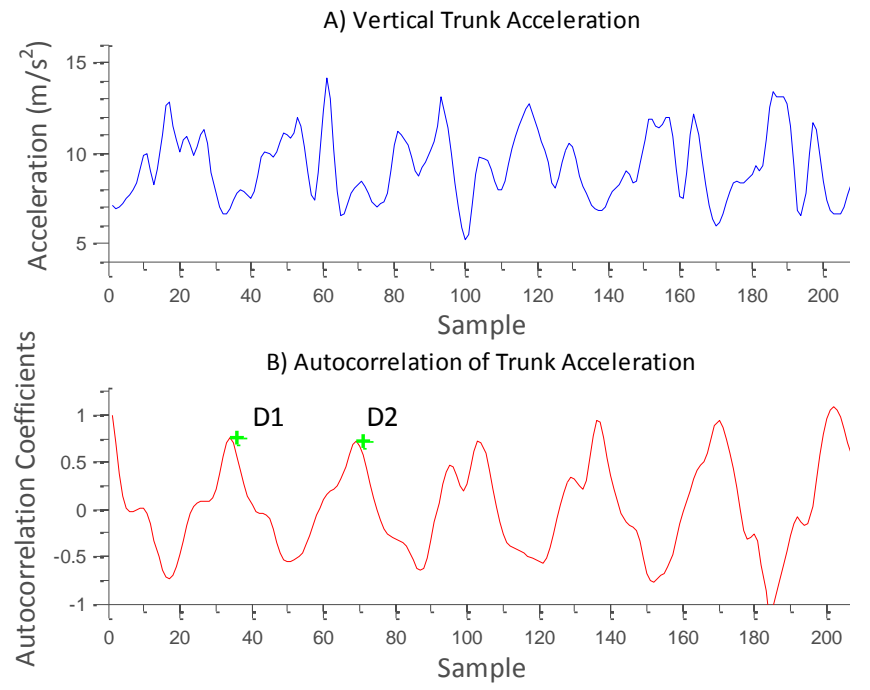

Figure 1. (a) A 3.5 second time-window of vertical trunk accerleration; and (b) the corresponding autocorrelation waveform.

autocorrelation is performed on this window every $200 \mathrm{~ms}$. Autocorrelation of vertical trunk acceleration provides two dominant peaks (Fig. 1). The first peak (D1) represents the previous step, and the second peak (D2) represents two steps back. By taking the ratio of the time between each peak, temporal symmetry can be found:

$$
\text { Temporal Symmetry }=\frac{n D 1}{n D 2-n D 1} \text { or } \frac{n D 2-n D 1}{n D 1}
$$

Where $n D 1$ is the time to the first dominant peak, and $n D 2$ is the time to the second dominant peak. For convention, gait symmetry values are presented as a ratio between 0 and 1 , where 1 represents perfect symmetry [17]. To stabilize the output of this symmetry calculation, a 5-width median filter was used before outputting a final symmetry value.
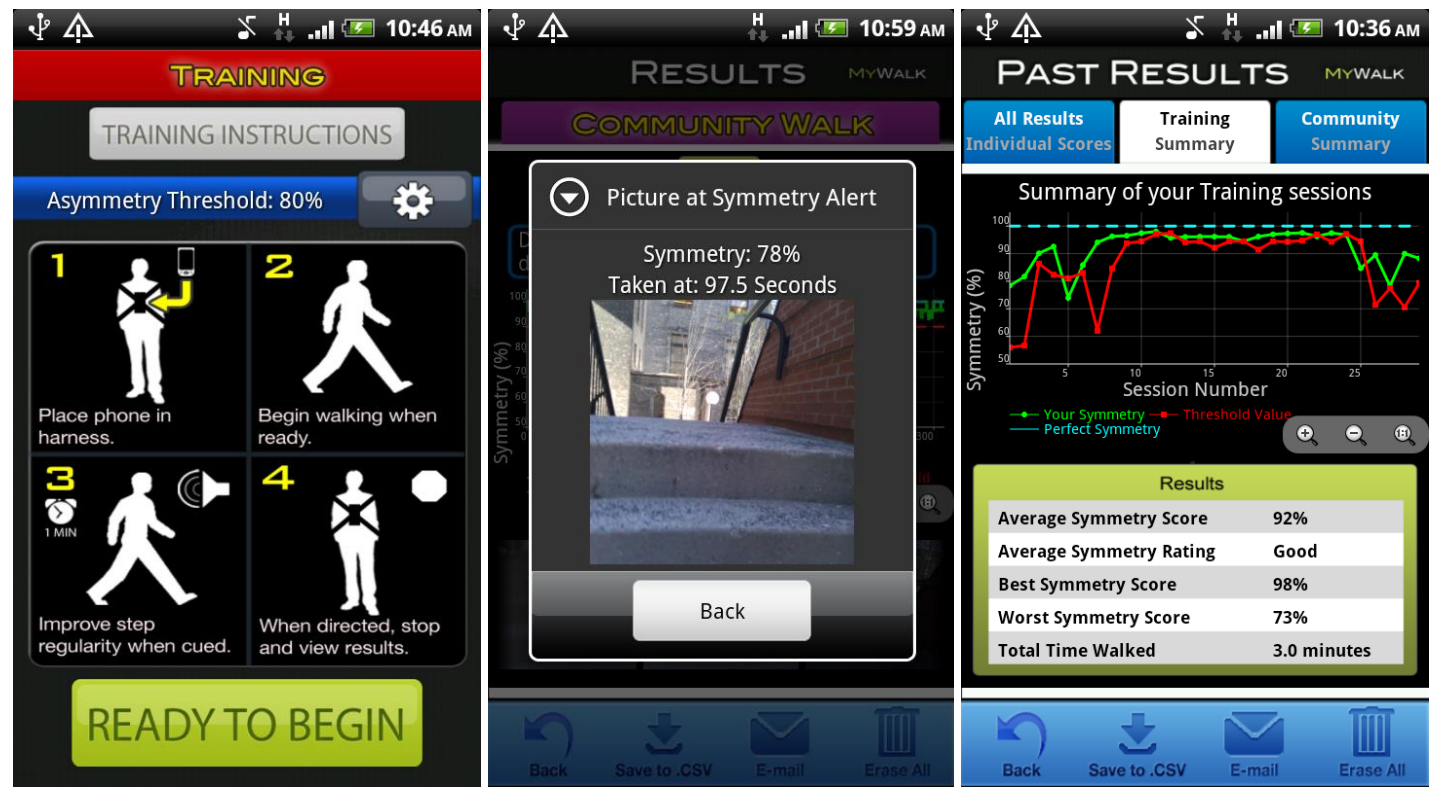

Figure 2. (a) [left] Instructions screen for MyWalk training mode; (b) [middle] Community walk mode takes a picture when step-time asymmetry falls below a set threshold; (c) [right] A summary of previous training sessions. 


\section{Training Mode}

In this mode, a user can improve their temporal symmetry through short one minute walking exercises (Fig. 2a). During training, MyWalk will assess the user's STA in real-time and give biofeedback prompts (auditory and/or vibration) if his/her STA falls below a certain threshold value. This biofeedback is meant to remind the user to walk more symmetrically. The threshold value can either be set automatically (from previous training results) or manually. Additionally, at the end of training the user can view a summary of his/her exercise results.

\section{Community Walk Mode}

The community walk has a similar operation to the training mode, except there is no time limit or biofeedback. Biofeedback was excluded due to the concerns of dual-tasking (i.e., exposure to two tasks simultaneously) in uncontrolled environments. Instead, when the user's STA falls below the threshold, a picture is taken with a timestamp of the exact STA at that moment (Fig. 2b). This mode is exploratory, yet could potentially help patients identify environments that adversely affect their walking patterns. This in turn could inform future therapy with their therapists.

\section{E. Progress History Mode}

Within this mode, a user can access previous results from training or community walks. As well, summary data to show a user's progress can be displayed (Fig. 2c). Users have the ability to share these data with their therapist via e-mail. This benefits the rehabilitation process by allowing therapists to monitor how often a patient exercises and how their STA is changing over time. Also by seeing their progress, a patient may have greater adherence to outpatient therapy.

\section{STA VALIDATION STUDY}

\section{A. Methods}

An initial validation study, with a healthy adult male volunteer, was completed to investigate the accuracy of MyWalk's STA calculation versus a ground truth measure of STA. The objective of this study was to evaluate if MyWalk could accurately monitor STA within different environments. A healthy adult was used due to convenience and a need for rapid evaluation. For future studies MyWalk's algorithm will be evaluated by members of the stroke population.

MyWalk was tested in three walking environments under two different walking scenarios: normal walking and induced asymmetric walking. The three walking environments were: (1) a 40m indoor ceramic floor hallway; (2) descending a $10 \mathrm{~m}$ outdoor ramp ( $15^{\circ}$ incline); and (3) a $20 \mathrm{~m}$ outdoor sidewalk. In total, there were six trials. Within the same trials, ground truth measures were obtained using a Delsys ${ }^{\circledR}$ Myomonitor ${ }^{\circledR}$ data logger attached to foot-switches (force-sensing resistors) in order to determine the exact time steps occurred (i.e., heelstrikes). From these step times, a ground truth value of temporal symmetry was calculated using a similar method to (1). Additionally, an accelerometer was attached to the Delsys ${ }^{\circledR}$ Myomonitor ${ }^{\circledR}$ data logger to time-sync the ground truth data with the STA output from MyWalk.
After data collection, all STA measures from MyWalk and the ground truth were interpolated to have the same sampling frequency. This was to allow for a distance/error value to be calculated between the two signals. The sampling frequency was set high, at $600 \mathrm{~Hz}$, to ensure reconstruction of the signals.

\section{B. Analsysis}

Root mean square error (RMSE) was used to compare the difference between MyWalk's STA and the ground truth values. RMSE can be determined by:

$$
R M S E=\sqrt{\frac{\sum_{t=1}^{n}\left(y_{t}-\hat{y}_{t}\right)^{2}}{n}} * 100 \%
$$

Where $n$ is the total number of samples, $t$ is the current sample, $y$ is the ground truth signal, and $\hat{y}$ is the experimental signal.

\section{RESULTS}

Table 1 summarizes the error values for all six trials. For normal walking RMSE ranged from 2.20-2.67\%, whereas for asymmetric walking RMSE ranged from 13.82-16.34\%. Fig. 3 shows examples of the signal waveforms that were used in the error calculations.

\section{TABLE I. ERROR IN MYWALK SYMMETRY OUTPUT}

\begin{tabular}{|c|l|l|}
\hline \multicolumn{2}{|c|}{ Walking Scenario } & RMSE (\%) \\
\hline \multirow{2}{*}{ Indoor Hallway } & Normal & 2.23 \\
\cline { 2 - 3 } & Asymmetric & 16.34 \\
\hline \multirow{2}{*}{ Outdoor Ramp } & Normal & 2.20 \\
\cline { 2 - 3 } & Asymmetric & 13.82 \\
\hline \multirow{2}{*}{ Outdoor Sidewalk } & Normal & 2.67 \\
\cline { 2 - 3 } & Asymmetric & 14.67 \\
\hline
\end{tabular}

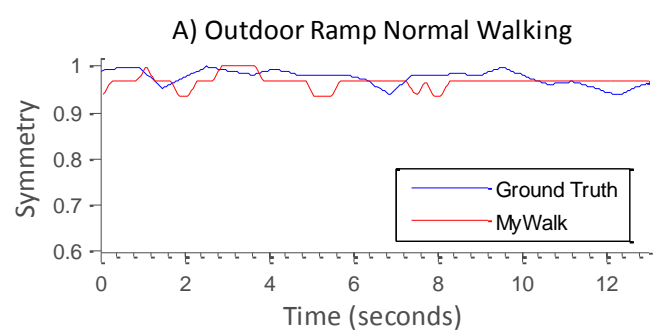

B) Outdoor Ramp Asymmetric Walking

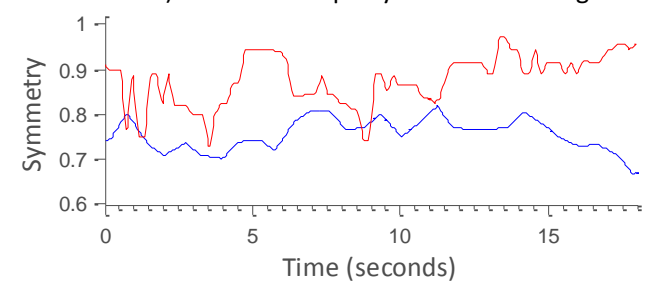

Figure 3. Comparison of STA output from ground truth foot-switches and MyWalk. Outdoor ramp environment in: (a) normal walking condition; and (b) asymmetric walking condition. 


\section{DISCUSSION}

\section{A. Study Results}

MyWalk achieved good accuracy ( $<5 \%$ RMSE) when monitoring STA in normal walking conditions. However, for asymmetric walking conditions the RMSE increased significantly (>10\% RMSE). Upon closer analysis of the STA waveforms in asymmetric walking conditions, it was found that MyWalk underestimates the severity of temporal asymmetry (Fig. 3b). This is likely attributed to a misdetection of autocorrelation peaks from asymmetric trunk acceleration. When a signal is less periodic (which is the case for asymmetric trunk acceleration compared to symmetric trunk acceleration), then it is harder to obtain strong dominant peaks from autocorrelation. Since MyWalk's STA is calculated from the location of autocorrelation's dominant peaks, failure to find these values will result in errors for STA. Other sources of error can be attributed to the fact that MyWalk is estimating steps from vertical trunk motion, versus actual step times. Nevertheless, when gait is in steady-state and at a constant velocity, the duration of each sub-component (or phase) of the gait cycle should be fairly consistent stride-to-stride. Upcoming work will attempt to improve the accuracy of MyWalk's STA monitoring. To do this, data from vertical trunk acceleration will be fed into MyWalk's algorithm with different parameters to see which will give the closest value to ground truth STA.

\section{B. Future Directions}

Future work will explore the novel use cases of MyWalk. Particularly, whether the community walk mode will be helpful to patients and clinicians in identifying environments that negatively affect walking symmetry, and whether MyWalk can be used as a tool for extended outpatient therapy.

This work will involve longer term, in-situ research studies, which are still lacking in literature [18]. MyWalk and future pervasive health technologies that enable gait rehabilitation in the context of everyday life require validation within realworld environments; this study was an initial step towards that validation process. Despite this challenge, these technologies will push forward the concept of contextualizing rehabilitation paradigms to their actual environment of use. By enabling this process, these technologies can be referred to as: contextual rehabilitation technologies.

Early discussions with physical therapists about the use of MyWalk have been promising. These therapists have mentioned a number of potential benefits with MyWalk, such as: enabling a stroke patient to self-monitor their rehabilitation progress, the ability to individualize training programs to a patient with the technology as a facilitator to patient adherence, and obtaining more information to assess outpatient walking in the context of a patient's everyday environment (e.g., pictures).

\section{CONCLUSION}

Advancements in wearable and pervasive computing technologies have opened up new doors for outpatient rehabilitation. MyWalk is an Android-based app that builds upon this notion. The goal of MyWalk is to conduct step-time asymmetry (STA) rehabilitation in the context of outpatient community settings. Initial validation results within different environments show that MyWalk performs well during symmetric walking conditions, however the accuracy of monitoring STA drops during asymmetric walking. Future work will be to improve the accuracy of MyWalk's STA monitoring, and to explore the utility of using MyWalk as a tool for outpatient rehabilitation. Contextual rehabilitation technologies, such as MyWalk, enable more opportunities to treat and assess patients in the context of their everyday environments. The implications of this technology-enabled contextualized approach to rehabilitation remains an area to be explored.

\section{REFERENCES}

[1] Public Health Agency of Canada, "Tracking heart disease and stroke in Canada," Canada, 2011.

[2] Canadian Institute of Health Information, "Factors predicting discharge home from inpatient rehabilitation after stroke," Canada, 2009.

[3] R. W. Bohannon, A. W. Andrews, and M. B. Smith, "Rehabilitation goals of patients with hemiplegia," Int. J. of Rehabilitation Research, vol. 11, no. 2, pp. 181-184, 1988.

[4] D. U. Jette et al., "Physical therapy interventions for patients with stroke in inpatient rehabilitation facilities," Physical Therapy, vol. 85, no. 3, pp. 238-248, 2005.

[5] K. K. Patterson, "Gait Asymmetry Post-Stroke," Ph.D. dissertation, Dept. Rehabilitation Sciences, Univ. Toronto, Toronto, ON, Canada, 2010.

[6] K. K. Patterson et al., "Gait asymmetry in community-ambulating stroke survivors," Archives of Physical Medicine and Rehabilitation, vol. 89, no. 2, pp. 304-310, 2008.

[7] R.A. States, E. Pappas, and Y. Salem, "Overground physical therapy gait training for chronic stroke patients with mobility deficits," Stroke, no. 3, 2009.

[8] K. J. McCain, P. S. Smith, F. E. Polo, S. C. Coleman, and S. Baker, "Excellent outcomes for adults who experienced early standardized treadmill training during acute phase of recovery from stroke: a case series," Topics in Stroke Rehabilitation, vol. 18, no. 4, pp. 428-436, 2011.

[9] D. Rose et al., "Feasibility and effectiveness of circuit training in acute stroke rehabilitation," Neurorehabilitation and NeuralRrepair, vol. 25 , no. 2 , pp. 140-148, 2011.

[10] F. Lowry, "Stroke rehabilitation services inadequate, experts say," Canadian Medical Assoc. J., vol. 182, no. 7, pp. E283-E284, 2010.

[11] K. K. Patterson, W. H. Gage, D. Brooks, S. E. Black, and W. E. McIlroy, "Changes in gait symmetry and velocity after stroke: a cross-sectional study from weeks to years after stroke," Neurorehabilitation and Neural Repair, vol. 24, no. 9, pp. 783-790, 2010.

[12] J. H. Carr and R. B. Shepherd, "Enhancing Physical Activity and Brain Reorganization after Stroke," Neurology Research Int., vol. 2011, 2011

[13] M. McCue, A. Fairman, and M. Pramuka, "Enhancing quality of life through telerehabilitation," Physical Medicine and Rehabilitation Clinics of North America, vol. 21, no. 1, pp. 195-206, 2010.

[14] J. Maitland, M. McGee-Lennon, and M. Mulvenna, "Pervasive healthcare: from orange alerts to mindcare," ACM SIGHIT Rec., vol. 1, no. 1, pp. 38-40, 2011.

[15] H. K. Y. Chan et al., "Feasibility study on iPhone accelerometer for gait detection," in Proc. th $^{\text {th }}$ In. Conf. on Pervasive Computing Technologies for Healthcare, 2011, pp. 184-187.

[16] M. Yang et al., "Assessing the utility of smart mobile phones in gait pattern analysis," Health Technology, vol. 2, pp. 81-88, 2012.

[17] C.-C. Yang, Y.-L. Hsu, K.-S. Shih, and J.-M. Lu, "Real-time gait cycle parameter recognition using a wearable accelerometry system," Sensors, vol. 11, no. 8, pp. 7314-7326, 2011.

[18] J. J. Kavanagh and H. B. Menz, "Accelerometry: a technique for quantifying movement patterns during walking," Gait \& Posture, vol. 28, no. 1, pp. 1-15, 2008. 